\title{
Angiogenesis in pituitary adenomas - relationship to endocrine function, treatment and outcome
}

\author{
H E Turner ${ }^{1}$, Zs Nagy $^{2,3}$, K C Gatter ${ }^{4}$, M M Esiri ${ }^{2}$, A L Harris ${ }^{5}$ \\ and J A H Wass ${ }^{\mathbf{1}}$ \\ ${ }^{1}$ Department of Endocrinology, Radcliffe Infirmary, Oxford, UK \\ ${ }^{2}$ Department of Neuropathology, Radcliffe Infirmary, Oxford, UK \\ ${ }^{3}$ Department of Pharmacology, University of Oxford, Oxford, UK \\ ${ }^{4}$ Department of Cellular Science, ICRF, Institute of Molecular Medicine, John Radcliffe Hospital, Oxford, UK \\ ${ }^{5}$ Department of Molecular Angiogenesis Group, ICRF, Institute of Molecular Medicine, John Radcliffe Hospital, Oxford, UK \\ (Requests for offprints should be addressed to J A H Wass, Department of Endocrinology and Metabolism, Nuffield Orthopaedic Centre, Windmill Road, \\ Oxford OX3 7LD, UK; Email: john.wass@noc.anglox.nhs.uk)
}

\begin{abstract}
Angiogenesis has been shown to be related to tumour behaviour, prognosis and response to treatment in many different tumour types. The aim of this study was to examine the relationship between angiogenesis and tumour behaviour and response to treatment in pituitary adenomas. The microvessel density (MVD) of pituitary tumours was assessed by counting blood vessels labelled with 3 different endothelial markers using antibodies to CD31, factor eight-related antigen and biotinylated Ulex europaeus (agglutinin I UEAI). One hundred and forty-two surgically removed pituitary adenomas (46 GH secreting, 6 microprolactinomas, 19 macroprolactinomas, $18 \mathrm{ACTH}$ secreting and 53 functionless tumours) were carefully characterized and assessed. There was a significant negative correlation between age and MVD of GH secreting tumours $\left(\mathrm{R}^{2}=33 \cdot 8, P=0 \cdot 005\right)$. Age was not
\end{abstract}

related to MVD in other tumour types. Pre-treatment hormone production by the adenomas was related to MVD in prolactinomas $(P<0 \cdot 05)$, but not in GH secreting tumours. Invasive prolactinomas were significantly more vascular than non-invasive tumours $(P<0 \cdot 05)$. Drug treatment with metyrapone or bromocriptine did not appear to influence tumour angiogenesis. Surgical cure was more likely in macroprolactinomas and in ACTH secreting tumours with lower MVD. These results show that factors related to angiogenesis are very important in determining a number of clinical features of pituitary tumours, in particular the invasiveness of macroprolactinomas, the effect of age in tumours secreting $\mathrm{GH}$ and the outcome of surgical treatment in macroprolactinomas and ACTH secreting tumours.

Journal of Endocrinology (2000) 165, 475-481

\section{Introduction}

Tumour growth beyond a few millimetres in diameter is angiogenesis dependent (Folkman 1990). In many human tumours, including breast, bladder and stomach, angiogenesis has been shown to be correlated with tumour behaviour (Weidner et al. 1991), outcome (Weidner et al. 1992, Bochner et al. 1995, Maeda et al. 1995) and response to therapy - the more vascular a tumour is, the lower the likelihood of any response to adjuvant therapy (Vacca et al. 1994, Gasparini et al. 1995, Hollingsworth et al. 1995).

The vascular density of different tumours has been assessed by counting vessels labelled with antibodies to different vascular endothelial markers including factor eight-related antigen (F8), CD31 (platelet endothelial cell adhesion molecule) and the lectin Ulex europaeus agglutinin 1 (UEAI). These markers have been shown to have different sensitivities for the detection of endothelium
(Holthofer et al. 1982, Horak et al. 1995). F8 stains large vessels but does not stain smaller microvessels (Mukai et al. 1980, Vermeulen et al. 1995), UEAI stains all microvessels and stains paraffin-embedded tissue well, but its drawback is that it stains the Golgi in some neoplastic cells (Holthofer et al. 1982, Witt \& Klessen 1987). CD31 is a sensitive marker for all microvessels but can be difficult to use in some paraffin-embedded tissue, possibly related to antigen loss due to fixatives containing acetic acid (Vermeulen et al. 1996).

We have recently shown that pituitary tumours are less vascular than the normal pituitary gland. We have shown that the vascular density of microprolactinomas is significantly less than macroprolactinomas, whereas there is no difference in the vascular density of microadenomas secreting growth hormone $(\mathrm{GH})$ when compared with GH secreting macroadenomas (Turner et al., 2000a). In this paper, we analysed the relationships between tumours 
arising from different pituitary hormone producing cells and angiogenesis. It is known that different types of pituitary tumours behave in different ways, for example silent corticotroph tumours are often more aggressive than other functionless tumours (Kovacs \& Horvath 1986). Furthermore, because it is known that older patients with acromegaly have smaller tumours and have milder disease with lower GH levels than patients of a younger age at presentation, its relationship to microvascular density (MVD) was analysed (Klijn et al. 1980, Smals et al. 1988). Finally, in view of the relationship in other tumours of angiogenesis with tumour behaviour and outcome, these factors were assessed in terms of tumour characteristics at presentation, drug treatment and surgical results.

\section{Materials and Methods}

\section{Specimen collection}

One hundred and forty-two surgically removed pituitary adenomas were investigated. There were $46 \mathrm{GH}$ secreting tumours (28 macroadenomas and 18 microadenomas), 6 microprolactinomas, 19 macroprolactinomas, 18 adrenocorticotrophin (ACTH) secreting tumours (Cushing's disease) and 53 non-functioning pituitary adenomas (29 gonadotroph, 21 negative and 3 silent ACTH). All the tissue had been fixed in $4 \%$ buffered formalin, dehydrated and embedded in paraffin. Histological examination and immmunohistochemistry for anterior pituitary hormones had been performed previously, and together with the clinical, endocrine and radiological data were used to characterise fully each tumour type. Invasiveness was defined according to the modified Hardy criteria (Bates et al. 1997). Tumours with evidence of bony destruction, spread into the sphenoid and/or cavernous sinus, or tumours with central nervous sytem/extracranial spread on computed tomography/magnetic resonance imaging were defined as invasive. 'Cure' was defined as an undetectable post-operative cortisol value in the patients with Cushing's disease, as a prolactin value in the normal range for patients with prolactinomas, and as a mean GH value of less than $5 \mathrm{mU} / 1$ or suppression to less than $2 \mathrm{mU} / 1$ on an oral glucose tolerance test for patients with acromegaly.

\section{Immunohistochemistry for CD31, UEAI and F8}

The streptavidin-biotin peroxidase complex technique $(\mathrm{ABC})$ was used for CD31 and F8, and the alkaline phosphatase/anti-alkaline phosphatase (APAAP) method was used for UEAI.

Sections $(4 \mu \mathrm{m})$ were mounted on aptes (3-aminopropyl triethoxy silane, Sigma, Poole, Dorset, UK)-coated slides, dewaxed and rehydrated. Endogenous peroxidase activity was blocked using 3\% hydrogen peroxide for CD31 and F8 antibodies. CD31 and F8 labelling required trypsinisation $(0 \cdot 1 \%$ trypsin in calcium buffer $)$ at $37^{\circ} \mathrm{C}$ for 15 min. CD31 labelling required further microwave pre-treatment in sodium citrate buffer $\mathrm{pH}$ 6. Labelling with UEAI did not require pre-treatment.

Non-specific primary antibody binding was blocked using fetal calf serum at a dilution of 1:20 for CD31 and UEAI. The primary antibodies were all applied for $60 \mathrm{~min}$ at room temperature, and washed three times in buffered saline. For CD31, the DAKO antibody was applied at a dilution of 1:20. An enhanced peroxidase one step (EPOS, DAKO, High Wycombe, Bucks, UK) F8 was applied as supplied. The biotinylated UEAI (Vector Laboratories, Burlingame, CA, USA) was used at a dilution of 1:200. An anti-mouse biotinylated secondary antibody (Insight Biotechnology, Wembley, Middlesex, UK) was applied at 1:200 dilution for $30 \mathrm{~min}$ at room temperature to the CD31 cases. This was followed by washes, and then application of the horseradish peroxidase streptavidin complex (DAKO) at 1:400 dilution for $30 \mathrm{~min}$. The APAAP complex (Vector Laboratories) was applied to the UEAI cases for $30 \mathrm{~min}$ followed by washes. Colour development was with metal enhanced diaminobenzidine (DAB) (Pierce and Warriner, Rockford, IL, USA) applied for 15 min to the F8 and CD31 cases, and with fast red substrate (Vector Laboratories) applied for $20 \mathrm{~min}$ to the UEAI cases. The slides were lightly counterstained with haematoxylin.

\section{Assessment of vascular density}

Vascular density was assessed by one examiner without knowledge of the tumour type or size. The Chalkley point technique was used as previously described (Fox et al. 1995, Turner et al. 2000a). An overall subjective semiquantitative grading system $(G)$ was also used (1 and 2 low and low moderate, and 3 and 4 - high and very high vascular density). The counts $(\mathrm{M})$ and grades $(\mathrm{G})$ were made by a single observer (H E T), and 20\% were checked by a second blinded observer (K G C). We have previously shown good intra-observer reliability for both Chalkley counts and overall grades (Turner et al. 2000a).

\section{Statistical analysis}

The Statgraphics software package was used. ANOVA was used for categorical data analysis and regression analysis for continuous variables.

\section{Results}

Patient characteristics - age, sex

There was no relation between patient age and vascular density of functionless tumours, macroprolactinomas and tumours causing Cushing's disease, but there was a 


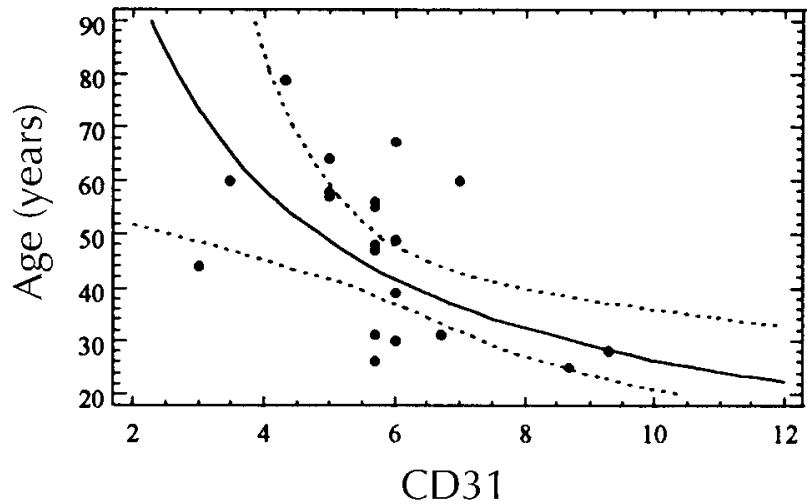

Figure 1 Relationship between age and vascular density in acromegaly. Axis $x$ : vascular density as measured by CD31 expression - mean Chalkley counts; axis y: age of the patients in years. Continuous line indicates best fitting regression curve (reciprocal $-Y$ model $-y=1 /(a+b x)$ ); dotted lines indicate $95 \%$ confidence levels.

significant negative correlation between age and vascular density in $\mathrm{GH}$ secreting tumours $\left(\mathrm{R}^{2}=22 \cdot 5 \%, P=0 \cdot 017\right.$ (UEAIM), $\mathrm{R}^{2}=33 \cdot 8 \%, P=0.005$ (CD31 M)) (Fig. 1). When the relationship between age and vascular density of patients with $\mathrm{GH}$ secreting macroadenomas and microadenomas was analysed separately, $\mathrm{R}^{2}=21 \cdot 2 \%$ and $73 \%$ respectively.

The sex of the patient did not affect vascular density of the different tumours (Table 1). An exception was prolactinomas where the patients with microprolactinomas were all women, and microprolactinomas were significantly less vascular than macroprolactinomas (14 males) $(P<0 \cdot 05)$.

\section{Hormone level and tumour type}

The pre-operative prolactin level from both microprolactinomas and macroprolactinomas was positively correlated with vascular density using both CD31 and UEAI $\left(\mathrm{R}^{2}=61 \cdot 3 \%, P=0 \cdot 003\right.$ (CD31), $\mathrm{R}^{2}=60 \cdot 6 \%, P=0 \cdot 0001$ (UEAI)) (Fig. 2). This relationship persisted when

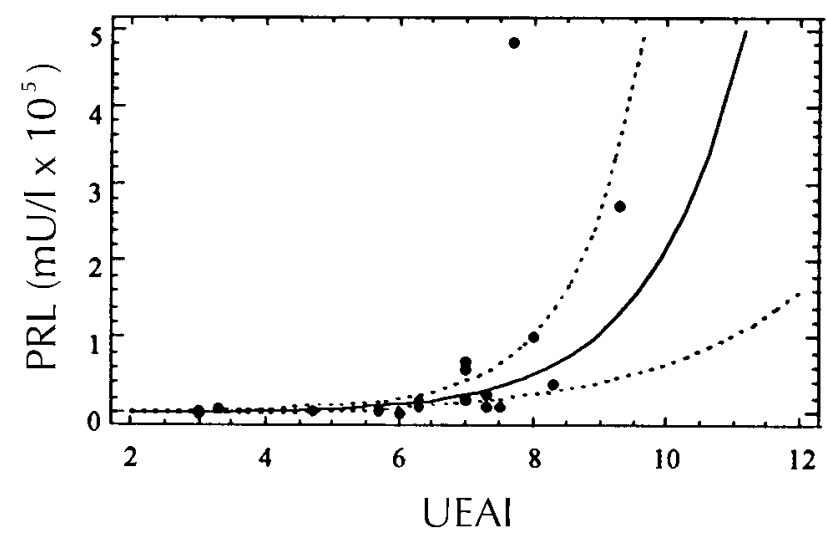

Figure 2 Relationship between preoperative serum prolactin levels and vascular density in prolactinomas. Axis $x$ : vascular density as measured by UEAI staining - mean Chalkley counts; axis $y$ : serum prolactin in $\mathrm{mU} / \mathrm{l}$. Continuous line indicates best fitting regression curve (exponential model $-y=e^{(a+b x)}$; dotted lines indicate $95 \%$ confidence levels.

pre-operative prolactin and vascular density in macroprolactinomas alone were considered $\left(\mathrm{R}^{2}=35 \cdot 6 \%, P=0 \cdot 02\right)$. Cortisol and ACTH production (assessed using urinary free cortisol (UFC) and ACTH levels) were not related to vascular density of tumours causing Cushing's disease (Table 2). Pre-operative GH (mean across an oral glucose tolerance test or random) and age-related insulin-like growth factor-I were not related to vascular density of GH secreting tumours.

\section{Tumour subtype}

Tumours that stained for GH alone $(n=14)$ were more vascular using ulex than those staining for GH and PRL $(n=11)(P=0 \cdot 036)$ (Table 3, Fig. 3). Although there was a trend for functionless tumours that were negative on immunostaining to be more vascular than tumours that stained for gonadotrophins, this was not statistically significant (Table 3). Silent corticotroph adenomas were the least

Table 1 Relationship between sex and vascular density. Results are means (S.E.M.). Numbers of cases in square brackets

\begin{tabular}{|c|c|c|c|c|c|c|}
\hline & CD31M & CD31G & F8M & F8G & UEAIM & UEAIG \\
\hline NFA-M & $5 \cdot 4(0 \cdot 4)[26]$ & $2 \cdot 6(0 \cdot 1)[26]$ & $5 \cdot 1(0 \cdot 5)[33]$ & $2 \cdot 3(0 \cdot 2)[33]$ & $7 \cdot 5(0 \cdot 8)[32]$ & $3 \cdot 0(0 \cdot 2)[32]$ \\
\hline NFA-F & $5.4(0.9)[8]$ & $2 \cdot 8(0 \cdot 5)[8]$ & $5 \cdot 3(1 \cdot 0)[10]$ & $2 \cdot 4(0 \cdot 5)[10]$ & $6 \cdot 9(1 \cdot 2)[10]$ & $2 \cdot 8(0 \cdot 4)[10]$ \\
\hline CUSH-M & & & $4 \cdot 7(0 \cdot 8)[4]$ & $1.9(0.6)[4]$ & $4.8(1.9)[4]$ & $2 \cdot 2(0 \cdot 5)[4]$ \\
\hline CUSH-F & & & $4 \cdot 7(0 \cdot 9)[10]$ & $1 \cdot 8(0 \cdot 4)[14]$ & $5 \cdot 1(0 \cdot 9)[10]$ & $2 \cdot 3(0 \cdot 2)[11]$ \\
\hline GH-M & $5 \cdot 5(0 \cdot 4)[11]$ & $2 \cdot 5(0 \cdot 5)[12]$ & $4 \cdot 6(0 \cdot 3)[23]$ & $1.9(0 \cdot 7)[25]$ & $7 \cdot 0(0 \cdot 8)[8]$ & $3 \cdot 0(0 \cdot 5)[20]$ \\
\hline $\mathrm{GH}-\mathrm{F}$ & $5 \cdot 6(0 \cdot 4)[14]$ & $2 \cdot 4(0 \cdot 6)[16]$ & $4 \cdot 9(0 \cdot 3)[23]$ & $1.9(0 \cdot 8)[26]$ & $7 \cdot 4(0 \cdot 5)[17]$ & $2.5(0.5)[12]$ \\
\hline
\end{tabular}


Table 2 Vascular density and hormonal activity of tumours. Results are means (S.E.M.). Numbers of cases are in square brackets

\begin{tabular}{|c|c|c|c|c|}
\hline & F8M & F8G & UEAIM & UEAIG \\
\hline UFC > $1000 \mathrm{nmol} / 24 \mathrm{~h}$ & $5 \cdot 1(0 \cdot 5)[5]$ & $1 \cdot 8(0 \cdot 5)[6]$ & $5 \cdot 1(1 \cdot 8)[6]$ & $2 \cdot 3(0 \cdot 5)[6]$ \\
\hline UFC $<1000 \mathrm{nmol} / 24 \mathrm{~h}$ & $4 \cdot 0(1 \cdot 4)[6]$ & $1 \cdot 7(0 \cdot 6)[8]$ & $5 \cdot 0(1 \cdot 0)[5]$ & $2 \cdot 1(0 \cdot 2)[6]$ \\
\hline $\mathrm{ACTH}>50 \mathrm{ng} / \mathrm{ml}$ & $5 \cdot 4(0 \cdot 8)[4]$ & $2 \cdot 2(0 \cdot 7)[5]$ & $5 \cdot 4(1 \cdot 2)[5]$ & $2 \cdot 3(0 \cdot 4)[5]$ \\
\hline АCTH <50 ng/ml & $4 \cdot 0(1 \cdot 3)[7]$ & $1 \cdot 5(0 \cdot 4)[10]$ & $4.5(0.8)[5]$ & $2 \cdot 0(0 \cdot 0)[6]$ \\
\hline
\end{tabular}

UFC, urinary free cortisol; F8M, mean Chalkley count using endothelial marker factor eight-related antigen; F8G, semiquantitative grade using endothelial marker factor eight-related antigen; UEAIM, mean Chalkley count using endothelial marker Ulex; UEAIG, semiquantitative grade using endothelial marker Ulex.

Table 3 Vascular density and tumour subtype. Results are means (S.E.M.). Numbers of cases are in square brackets

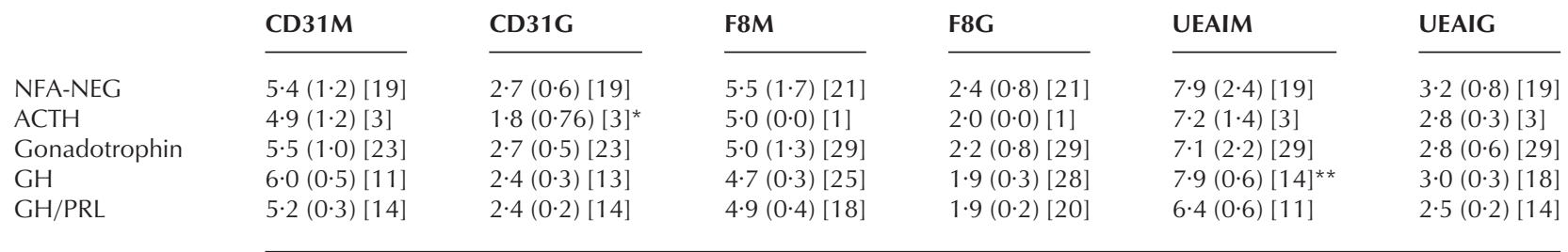

NEG, negative; NFA, non-functioning tumours; PRL, prolactin; CD31M, mean Chalkley count using endothelial marker CD31; CD31G, semiquantitative grade using endothelial marker CD31; F8M, mean Chalkley count using endothelial marker factor eight-related antigen; F8G, semiquantitative grade using endothelial marker factor eight-related antigen; UEAIM, mean Chalkley count using endothelial marker Ulex; UEAIG, semiquantitative grade using endothelial marker Ulex. ${ }^{*}$ ACTH significantly lower than tumours that were negatively (NEG) staining and gonadotrophin staining tumours, $P<0 \cdot 05$.

${ }^{* *} \mathrm{GH}$ staining tumours significantly more vascular than $\mathrm{GH} / \mathrm{PRL}$ staining tumours, $P=0 \cdot 036$.

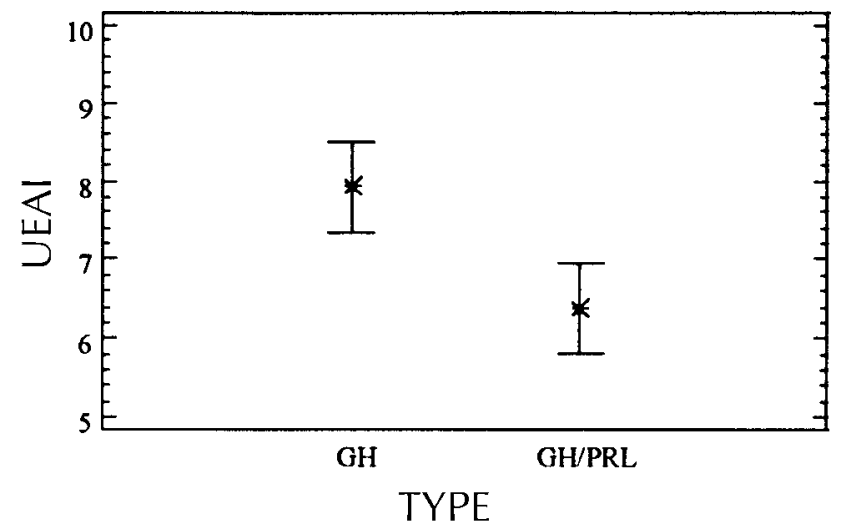

Figure 3 Vascular density in different types of growth hormone secreting tumours. UEAI: UEAI staining - mean Chalkley counts. $\mathrm{GH}$ : growth hormone immuno-positive tumours $(n=14)$; GH/PRL: growth hormone and prolactin immuno-positive tumours $(n=11)$. Asterisks indicate mean values; error bars indicate the standard error of the mean.

vascular type of functionless tumour using all vascular markers, although this only achieved statistical significance with CD31 $(P<0 \cdot 05)$.

\section{Tumour invasiveness}

This was defined according to the modified Hardy criteria (Bates et al. 1997). Invasive macroprolactinomas $(n=8)$

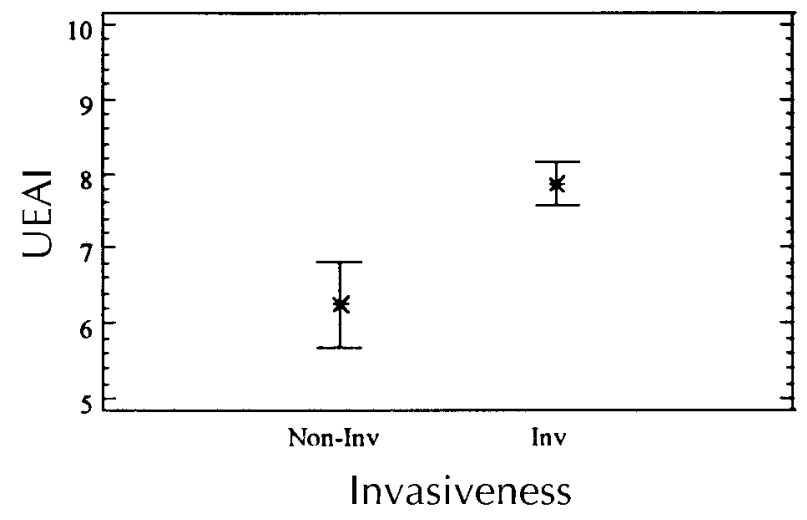

Figure 4 Vascular density in macroprolactinomas. UEAI: UEAI staining - mean Chalkley counts. Non-Inv: non-invasive macroprolactinomas $(n=7)$; Inv: invasive macroprolactinomas $(n=8)$. Asterisks indicate mean values; error bars indicate the standard error of the mean.

were significantly more vascular than non-invasive macroprolactinomas $(n=7)$ (Fig. 4) $(P<0.05$ ANOVA, $P=0.042 t$-test $)$. The vascular density of invasive macroprolactinomas reached that of the normal pituitary gland. There was no such difference in vascular density between invasive $(n=7)$ and non-invasive $(n=21) \mathrm{GH}$ secreting macroadenomas. Although invasive functionless tumours tended to be more vascular, the differences did not reach statistical significance. 
Table 4 Effect of treatment on vascular density. Results are means (S.E.M.). Numbers of cases are in square brackets

\begin{tabular}{|c|c|c|c|c|c|c|}
\hline & CD31M & CD31G & F8M & F8G & UEAIM & UEAIG \\
\hline Metyrapone & $3 \cdot 0(0 \cdot 0)[2]$ & & $4 \cdot 8(0 \cdot 6)[4]$ & $2 \cdot 0(0 \cdot 7)[4]$ & $5 \cdot 1(1 \cdot 7)[6]$ & $2 \cdot 3(0 \cdot 3)[6]$ \\
\hline No metyrapone & $3 \cdot 5(0 \cdot 0)[1]$ & & $4 \cdot 8(1 \cdot 0)[8]$ & $2 \cdot 0(0 \cdot 4)[9]$ & $4 \cdot 9(0 \cdot 7)[7]$ & $2 \cdot 1(0 \cdot 1)[7]$ \\
\hline DA Ag Res & $6 \cdot 2(0 \cdot 8)[4]$ & $3 \cdot 5(0 \cdot 5)[6]$ & $4 \cdot 7(1 \cdot 2)[9]$ & $2 \cdot 3(0 \cdot 4)[9]$ & $7 \cdot 5(0.5)[9]$ & $2 \cdot 8(0 \cdot 2)[9]$ \\
\hline DA Ag sensitive & $6 \cdot 4(0 \cdot 2)[2]$ & $3 \cdot 0(0 \cdot 0)[2]$ & $5 \cdot 4(0.9)[5]$ & $2 \cdot 4(0 \cdot 4)[5]$ & $6 \cdot 0(2 \cdot 0)[4]$ & $2 \cdot 4(1 \cdot 0)[4]$ \\
\hline Cushing's cure & $3 \cdot 0(0 \cdot 4)[2]$ & & $4.6(0.9)[11]$ & $1 \cdot 9(0 \cdot 3)[13]$ & $4 \cdot 3(0 \cdot 8)[8]^{*}$ & $2 \cdot 1(0 \cdot 1)[8]$ \\
\hline No cure & $3 \cdot 3(0 \cdot 0)[2]$ & & $5 \cdot 0(0 \cdot 9)[3]$ & $1 \cdot 7(0 \cdot 6)[5]$ & $6 \cdot 0(1 \cdot 3)[6]$ & $2 \cdot 4(0 \cdot 3)[7]$ \\
\hline Macroprolactinoma cure & $6 \cdot 3(0 \cdot 0)[1]$ & $3 \cdot 0(0 \cdot 0)[1]$ & $4 \cdot 4(1 \cdot 6)[2]$ & $2 \cdot 0(0 \cdot 0)[2]$ & $4 \cdot 8(3 \cdot 0)[2]^{*}$ & $1 \cdot 8(1 \cdot 6)[2]$ \\
\hline No cure & $6 \cdot 3(0.5)[8]$ & $3 \cdot 3(0 \cdot 4)[10]$ & $5 \cdot 0(0.5)[17]$ & $2 \cdot 4(0 \cdot 3)[17]$ & $7 \cdot 4(0 \cdot 6)[15]^{*}$ & $2 \cdot 8(0 \cdot 2)[16]$ \\
\hline $\mathrm{GH}$ cure & $5 \cdot 4(0 \cdot 5)[13]$ & $2 \cdot 4(0 \cdot 4)[13]$ & $4 \cdot 7(0.6)[22]$ & $1.9(0 \cdot 3)[24]$ & $7 \cdot 4(0 \cdot 5)[9]$ & $2 \cdot 7(0 \cdot 6)[14]$ \\
\hline No cure & $5.9(1.0)[9]$ & $2 \cdot 5(0 \cdot 5)[10]$ & $5 \cdot 4(1 \cdot 2)[11]$ & $2 \cdot 0(0 \cdot 5)[14]$ & $8 \cdot 1(0 \cdot 6)[10]$ & $3 \cdot 2(0 \cdot 5)[11]$ \\
\hline
\end{tabular}

DA Ag Res, prolactinomas removed from patients who were resistant to treatment with dopamine agonists; DA Ag sensitive, tumours that were removed from patients who were sensitive to dopamine agonists; $\mathrm{GH}$, GH secreting tumours causing acromegaly; CD31M, mean Chalkley count using endothelial marker CD31; CD31G, semiquantitative grade using endothelial marker CD31; F8M, mean Chalkley count using endothelial marker factor eight-related antigen; F8G, semiquantitative grade using endothelial marker factor eight-related antigen; UEAIM, mean Chalkley count using endothelial marker Ulex; UEAIG, semiquantitative grade using endothelial marker Ulex.

${ }^{*} P<0.05$ for difference between cure and no cure.

\section{Response to treatment}

Macroprolactinomas and tumours causing Cushing's disease that were cured by trans-sphenoidal surgery were significantly less vascular than those not cured by surgery (Table 4). There was no such association seen with GH secreting tumours.

Tumours removed from patients with Cushing's disease who were treated with metyrapone were of a similar vascular density to tumours in untreated patients (Table 4). There was no difference in MVD in prolactinomas removed from patients who had received treatment with bromocriptine (Table 4). Insufficient numbers of patients with acromegaly received somatostatin agonist treatment prior to surgery for any conclusions to be reached about the effect of octreotide on vascular density.

\section{Comparison of different vascular markers}

A comparison (Spearman rank correlation) of the three vascular markers showed that the grade and Chalkley mean using each marker - CD31, UEAI and F8 - were significantly related $\left(\mathrm{R}^{2}=82 \%, \mathrm{R}^{2}=92 \%\right.$, and $\mathrm{R}^{2}=90 \%$ respectively). Comparisons between the three markers showed that CD31 Chalkley mean and grade were significantly related to UEAI Chalkley mean and grade $(P<0.02, \mathrm{R}=0.31)$. However the Chalkley mean and grade measured using F8 were poorly related to the measurement using CD 31 and UEAI $(P>0 \cdot 05)$. The total counts using F8 were lower than those using CD31 and UEAI.

\section{Discussion}

Vascular counts determined using immunostaining for CD31 and UEAI were more sensitive than F8 and led to higher overall MVD. Although antibodies to CD31 are not completely specific for endothelial cells, as they may also detect plasma cells, this did not present any practical difficulties (Albelda et al. 1991). Ulex is a lectin that binds to fucose containing residues present on endothelial cells, and in some pituitary specimens ulex also stained the Golgi region, making vascular counting impossible (Witt \& Klessen 1987). All vascular endothelia do not appear to synthesise factor 8 related antigen (FVIIIAg) and so this marker does not stain all microvessels (Mukai et al. 1980). This may be related to differences in synthesis of F8. The good correlation shown between counts using CD31 and UEAI corresponds to the fact that these markers have been shown to stain the majority of tumour microvessels, despite the slight drawbacks mentioned above. The poor correlation between both these markers and F8 is in keeping with the more limited expression of this marker. However, despite these differences the results with each marker were similar although the total vascular counts differed. For future studies in the pituitary gland, either UEAI or CD31 are probably optimal, although differences in tissue preservation can make CD31 less straightforward to use.

Tumour microvascular density was clearly related to the age of the patient when tumours secreting GH were considered, fitting with the clinical observation that acromegaly is a milder disease with smaller tumours and lower GH levels in patients who present when older, and it is the younger patients with acromegaly who are likely to have the aggressive larger tumours (Klijn et al. 1980, Smals et al. 1988). This relationship between angiogenesis and age in $\mathrm{GH}$ secreting tumours is not related to size, as we have previously shown that the MVD of microadenomas and macroadenomas secreting GH is similar (Turner et al. 2000a), and also the relationship persisted when microadenomas and macroadenomas were analysed 
separately. It suggests that there may be a difference in angiogenic mechanisms in pituitary tumours from older patients. There are some data to suggest that angiogenesis is impaired with ageing (Rivad et al. 1999). Endothelial cells may become less responsive with age and vascular endothelial growth factor (VEGF) expression is reduced. There was no such statistically significant relationship observed between age and angiogenesis in other tumour types, although there was a trend in all tumour types for the adenomas removed from older patients to have lower MVD. This is in keeping with the observation that there is a trend to lower proliferation indices (measured using Ki-67) in pituitary tumours removed from older patients in comparison with younger patients (Yonezawa et al. 1997).

The association between vascular density and pretreatment prolactin concentration is related to the fact that macroprolactinomas are significantly more vascular than microprolactinomas (Turner et al., 2000a) and higher pre-treatment prolactin concentrations are found in cases with macroprolactinomas. Pre-treatment prolactin concentrations were related to angiogenesis when macroprolactinomas alone were considered. It is interesting to note that although the difference did not reach statistical significance, the vascular density of ACTH producing tumours associated with higher UFC and ACTH were consistently higher than the tumours associated with lower hormonal activity. This is in contrast to GH producing tumours where there is no relationship between tumour size and GH production, and we have shown that there is no relationship of vascular density with tumour size or hormonal production.

Tumours producing GH and prolactin were less vascular than the tumours producing $\mathrm{GH}$ alone. It is relevant to speculate whether tumour production of $21 \mathrm{kDa}$ prolactin also reflects production of $16 \mathrm{kDa}$ prolactin - a known inhibitor of angiogenesis (Clapp et al. 1993) and that this leads to a restraining influence on angiogenesis in these tumours. The low vascular density of the silent ACTH tumours is difficult to explain when their clinical behaviour is often aggressive. It suggests that there are other factors determining their behaviour, such as alterations in cell cycle control secondary to, for example, alterations in p27 expression (Lidhar et al. 1998). The results in functionless tumours are in contrast to those of Pawlikowski et al. (1997) who showed that tumour expression of follicle-stimulating hormone was associated with the highest vascular density; however, the total number of tumours studied in their paper was small $(n=22)$ and they did not study tumour hot spots (Pawlikowski et al. 1997). The differences were only present when vascular density using F8 and ulex were assessed.

A relationship between angiogenesis and tumour behaviour was demonstrated when invasiveness was considered. This was particularly apparent when invasive macroprolactinomas were considered, in keeping with the fact that these tumours tend to be the most aggressively invasive type of benign pituitary adenoma. Jugenburg and colleagues (1995) showed no difference between invasive and non-invasive tumours in terms of vascular density, although they did demonstrate that if the MVD of hot spots in pituitary carcinomas were assessed, the MVD was higher than in benign adenomas, suggesting an association between tumour behaviour and aggressiveness (Jugenburg et al. 1995). There is a biological problem in assessing invasiveness, as associations between tumour behaviour and invasiveness differ depending on the way that invasiveness is assessed - varying from microscopic examination of the dura to intra-operative observation or radiological assessment (Turner \& Wass 1999). Invasiveness of pituitary tumours has been shown in other studies to correlate with factors such as tumour proliferation index (Turner \& Wass 1999).

There was a significant relationship between angiogenesis and surgical cure in macroprolactinomas and ACTH secreting tumours, as those that were less vascular were more likely to be cured. This is likely to be related to differences in tumour invasiveness and difficulty in completely resecting more vascular tumours. Cure in functionless tumours is difficult to define as there is no hormonal product to use as a tumour marker, but we have shown that MVD cannot be used to predict which functionless tumours will regrow and recur (Turner et al., 2000b).

In Jugenburg's study, prolactinomas removed from patients treated with bromocriptine showed a trend to lower MVD than those removed from untreated patients, although these differences did not achieve statistical significance (Jugenburg et al. 1995). In our cohort of tumours, treatment of patients with ACTH secreting tumours with metyrapone, or those with prolactinomas with bromocriptine did not appear to be associated with altered vascular density. This is perhaps not surprising as these drugs are not known to inhibit angiogenesis per se. There are significant methodological problems with investigating the effects of these drugs as duration of treatment and compliance are difficult to assess. Finally, the results may be confounded by the administration of these medications to reduce hormonal levels because the tumours in these particular cases were more active and the associated clinical features more florid.

In conclusion, these results show that factors related to angiogenesis (measured using MVD) are very important in determining a number of known clinical features of pituitary tumours, in particular the invasiveness of macroprolactinomas, the effect of age in tumours secreting $\mathrm{GH}$ and the outcome of surgical treatment in macroprolactinomas and ACTH secreting tumours. It is possible that inhibitors of angiogenesis may be of value in recurrent prolactinomas and since radiotherapy is more effective in well-oxygenated tumours, that this mode of treatment should be used in more highly angiogenic cases. The relationship between angiogenesis and tumour invasiveness suggests, as shown in other tumour types, that 
angiogenesis increases in pituitary adenomas as they become more aggressive. It will be interesting to study MVD in the very rare cases of pituitary carcinoma to determine whether these tumours have an even higher MVD.

\section{References}

Albelda SM, Muller WA, Buck CA \& Newman PJ 1991 Molecular and cellular properties of PECAM (endoCAM/CD31): a novel vascular cell-cell adhesion molecule. Journal of Cell Biology 14 1059-1068.

Bates AS, Farrell WE, Bicknell EJ, McNicol AM, Talbot AJ, Broome JC, Perrett CW, Thaker RV \& Clayton RN 1997 Allelic deletion in pituitary adenomas reflects aggressive biological activity and has potential value as a prognostic marker. Journal of Clinical Endocrinology and Metabolism 82 818-824.

Bochner BH, Cote RJ, Weidner N, Groshen S, Chen SC, Skinner DG \& Nichols PW 1995 Angiogenesis in bladder cancer: relationship between microvessel density and tumour prognosis. Journal of the National Cancer Institute 87 1603-1612.

Clapp C, Martial JA, Guzman RC, Rentier-Delrue F \& Weiner RI 1993 The 16-kilodalton N-terminal fragment of human prolactin is a potent inhibitor of angiogenesis. Endocrinology 133 1292-1299.

Folkman J 1990 What is the evidence that tumours are angiogenesis dependent? Journal of the National Cancer Institute 82 4-6.

Fox SB, Leek RD, Weekes MP, Whitehouse RM, Gatter KC \& Harris AL 1995 Quantification and prognostic value of breast cancer angiogenesis: Chalkley count and computer image analysis. Journal of Pathology 177 275-283.

Gasparini G, Bevilacqua P, Bonoldi E, Testolin A, Galassi A \& Verderio P 1995 Predictive and prognostic markers in a series of patients with head and neck squamous-cell invasive carcinoma treated with concurrent chemoradiation therapy. Clinical Cancer Research 1 1375-1383.

Hollingsworth HC, Kohn EC, Steinberg SM, Rothenberg ML \& Merino MJ 1995 Tumour angiogenesis in advanced stage ovarian carcinoma. American Journal of Pathology 147 33-41.

Holthofer H, Virtanen I, Kariniemi AL, Hormia M, Linder E \& Miettinen A 1982 Ulex europaeus I lectin as a marker for vascular endothelium in human tissues. Laboratory Investigation 47 60-65.

Horak ER, Leek R, Klenk N, LeJeune S, Smith K, Stuart N, Greenall M, Stepniewska K \& Harris AL 1995 Angiogenesis, assessed by platelet/endothelial cell adhesion molecule antibodies, as indicator of node metastasis and survival in breast cancer. Lancet 340 $1120-1124$.

Jugenburg M, Kovacs K, Stefaneanu L \& Scheithauer BW 1995 Vasculature in nontumorous hypophyses, pituitary adenomas and carcinomas: a quantitative morphologic study. Endocrine Pathology 6 $115-124$.

Klijn JGM, Lamberts SWJ, de Jong FH, an-Dongen KJ \& Birkenhager JC 1980 Interrelationships between tumour size, age, plasma growth hormone and incidence of extra-sellar extension in acromegalic patients. Acta Endocrinologica 95 289-297.

Kovacs K \& Horvath E 1986 Tumours of the Pituitary Gland. Washington: Armed Forces Institute of Pathology.

Lidhar K, Korbonitis M, Khalimova Z, Jordan S, Lu X, Lowe DG \& Grossman AB 1998 Reduced expression of p27 immunoreactivity in pituitary tumours, particularly corticotroph adenomas and malignant tumours. Journal of Endocrinology 157 Abstract 20.

Maeda K, Chung Y-S, Takasuka S, Ogawa Y, Sawada T, Yamashita $\mathrm{Y}$ et al. 1995 Tumour angiogenesis as a predictor of recurrence in gastric carcinoma. Journal of Clinical Oncology 13 477-481.

Mukai K, Rosai J \& Burgdorf WHC 1980 Localisation of factor VIII-related antigen in vascular endothelial cells using an immunoperoxidase method. American Journal of Surgical Pathology 4 273-276.

Pawlikowski M, Pisarek H \& Jaranowska M 1997 Immunocytochemical investigations on the vascularisation of pituitary adenomas. Endocrine Pathology 8 189-193.

Rivad A, Fabre J, Silver M, Chen D, Murohara T, Kearney M, Magner M, Asahara T \& Isner JM 1999 Age-dependent impairment of angiogenesis. Circulation 99 111-120.

Smals AEM, Pieters GFFM, Smals AGH \& Kloppenborg PWC 1988 Sex difference in the relation between sellar volume and basal and GH-releasing hormone stimulated GH in acromegaly. Acta Endocrinologica 117 387-391.

Turner HE \& Wass JAH 1999 Are markers of proliferation useful in the histological assessment of pituitary tumours? Pituitary 1 147-151.

Turner HE , Nagy ZS, Gatter KC, Esiri MM, Harris AL \& Wass JAH 2000a. Angiogenesis in pituitary adenomas and the normal pituitary gland. Journal of Clinical Endocrinology and Metabolism $\mathbf{8 5}$ (In Press).

Turner HE, Nagy ZS, Gatter KC, Esiri MM, Harris AL \& Wass JAH 2000b. Proliferation, Bcl-2 expression and angiogenesis in pituitary. British Journal of Cancer (In Press).

Vacca A, Ribatti D, Roncati L, Ranier G, Serio G, Silvestrini F \& Dammacco F 1994 Bone marrow angiogenesis and progression in multiple myeloma. British Journal of Haematology 87 503-508.

Vermeulen PB, Verhoeven D, Fierens H, Hubens G, Goovaerts G, Van-Marck E, De Bruijn EA, Van Oosterom AT \& Dirix LY 1995 Microvessel quantification in primary colorectal carcinoma: an immunohistochemical study. British Journal of Cancer 71 340-343.

Vermeulen PB, Gasparini G, Fox SB, Toi M, Martin L, McCulloch P, Pezella F, Viale G, Weidner N, Harris AL \& Dirix LY 1996 Quantification of angiogenesis in solid human tumours: an international consensus on the methodology and criteria of evaluation. European Journal of Cancer 32A 2474-2484.

Weidner N, Semple JP, Welch WR \& Folkman J 1991 Tumour angiogenesis and metastasis: correlation in invasive breast carcinoma. New England Journal of Medicine 324 1-8.

Weidner N, Folkman J, Pozza F, Bevilacqua P, Allred EN, Moore DH, Meli S \& Gasparini G 1992 Tumour angiogenesis: a new significant and independent prognostic indicator in early stage breast carcinoma. Journal of the National Cancer Institute 84 1875-1887.

Witt M \& Klessen CH 1987 Galactose and fucose binding sites in anterior pituitary cells of the rat: detection by means of biotinylated lectins. Folia Histochemica et Cytobiologica 25 115-118.

Yonezawa K, Tamakai N \& Kokunai T 1997 Clinical features and growth fractions of pituitary adenomas. Surgical Neurology 48 494-500.

Received 11 October 1999

Accepted 30 December 1999 